\title{
IdleWars: an Evaluation of a Pervasive Game to Promote Sustainable Behaviour in the Workplace
}

\author{
Evangelos Tolias ${ }^{1}$, Enrico Costanza ${ }^{1}$, Alex Rogers ${ }^{1}$, Benjamin Bedwell ${ }^{2}$, Nick Banks ${ }^{3}$ \\ 1 Electronics and Computer Science, University of Southampton, Southampton SO171BJ, \\ \{et2e10, ec, acr\}eecs.soton.ac.uk \\ 2 Horizon Digital Economy Research, University of Nottingham, Nottingham, NG72TU, \\ Benjamin. Bedwellenottingham.ac.uk \\ ${ }^{3}$ Centre for Sustainable Energy, Bristol BS34AQ, \\ Nick.Banks@cse.org.uk
}

\begin{abstract}
Energy reduction is one of the main challenges that countries around the world currently face, and there is potential to contribute to this by raising awareness towards sustainability in the workplace. We introduce IdleWars, a pervasive game played using smartphones and computers. In the game, workers' proenvironmental or wasteful behaviour is reflected in their game score, and displayed through eco-feedback visualisations to try and call attention to energy wastage and potentially reduce it. A field deployment, over two weeks in a medium sized organisation, revealed that the physical and competitive elements of the game work well in engaging participants and stimulating discussion around energy wasted and conservation. However, the game turned out to encourage also some anti-conservation behaviours, as participants appropriated the game and extended its rules, sometimes in a way that favoured engagement and fun rather than proenvironmental behaviour. More in general, our study uncovered how both the game and idle time reduction in itself can rub against the daily practices of the workplace where the study was run.
\end{abstract}

\section{Introduction}

Energy reduction is one of the main challenges that countries around the world are currently facing, and it has been pointed out that there is an important energy saving potential in the work environment [11,4]. A large part of this potential is related to energy wastage $[9,19]$, i.e. equipment being left on when not in use. As an example, according to the Personal Computer (PC) energy report [14], in the UK 27\% of the workers who regularly use a PC reported that they do not always shut down their computers, $14 \%$ reported that they shut them down only occasionally, while $9 \%$ reported to never shut them down. This energy wastage results not only in additional cost for the industry, but, more importantly, in unnecessary carbon emissions.

We refer to the time that a computer is left on while not in use as "idle time", and we argue that idle time reduction offers a rich opportunity to study interventions to promote behaviour change in the work place. It is worth emphasising that the aim of this work is researching behaviour change interventions, rather than directly addressing reduction of computer energy consumption. Indeed, there are already commercial 
solutions available on the market to reduce computer idle time by automatically switching computers off at predefined times (e.g. 1E NightWatchman ${ }^{4}$, Cisco EnergyWise Suite $^{5}$ ). Adopting computer idle time ${ }^{6}$ reduction as a research vehicle is useful because it provides a convenient scenario (as detailed below) to design and evaluate behaviour change intervention, which could then be applied to other forms of resources wastage.

In this paper we present an approach that combines a game with eco-feedback [5] in the work environment. We introduce IdleWars, a pervasive game played using smartphones and desktop computers. In our game, workers' proenvironmental or wasteful behaviour is reflected in their game score, and displayed through eco-feedback visualisations. Our aim is to use a game as an engagement mechanism to activate intrinsic motivations [17], and to try and bring the workers' attention to their computer based energy wastage and reduce it.

The rest of this paper is structured as follows. The next section presents a brief discussion of related work around eco-feedback and games related to sustainability. Subsequently, we introduce the design of IdleWars and its rationale, followed by its technical implementation. We conclude by reporting an initial two-week deployment of the game in a real workplace, which demonstrates that workers immediately grasped the game design and engaged with it.

\section{Related Work}

Froehlich et al. [5] provide a review of eco-feedback technology and interventions, discussing the potential benefits of cooperation between the academic fields of HCI and environmental psychology. More specifically, they argue that HCI design for proenvironmental behaviour change can be informed by models (e.g. rational choice models, norm activation models) and strategies already popular in environmental psychology (e.g. information, goal setting, comparison). To date, research related to eco-feedback is mostly focused on the domestic environment [5]. For example, Jain et al. [10] investigated visualisations based on consequence interventions like comparative feedback, historical feedback (current day, last week), rewards and penalties. Their results show that there is a link between interface engagement and energy conservation.

Few papers have investigated eco-feedback in the work environment. Siero et al. [19] investigated behaviour change related to energy conservation in two units of a metallurgical company. The first unit received feedback and goal setting interventions, whilst the second unit additionally received comparative feedback (its performance was compared to that of the first unit). Their analysis suggest that employing comparative feedback results in higher energy conservation. Pousman et al. [15] proposed Imprint, a system that tracks the documents people print in the work environment, and provides a visualisation of the resources consumed in this way on a semi-public display. The authors suggest that their design employs a "ludic engagement strategy" [6] to stress environmental issues related with energy and paper consumption. Schwartz et al. [18] installed plug-level energy meters in a few offices of a research organisation and observed the reactions of

\footnotetext{
${ }^{4}$ http://www.1e.com/nightwatchman-pc-power-management/

${ }^{5}$ http://www.cisco.com/en/Us/products/ps10195/

${ }^{6}$ A typical desktop PC and LCD display consume $55 \mathrm{~W}$ and $15 \mathrm{~W}$ of power respectively.
} 
employees through business ethnography. They claim that the sensors enabled workers to be aware of their own energy related behaviour in the workplace. Jentsch et al. [11] presented an energy-saving support system for work environments that leverages a variety of sensors (temperature, electricity, light, contact) to provide workers suggestions about how to act in an environmentally friendly way, however, no real-world evaluation is reported. Yun et al. [21] run a twenty-seven week field study with eighty employees investigating feedback interventions combined with manual and automatic control. The manual control enables participants to activate and deactivate a device remotely via a web interface whereas automatic control is a web-based scheduler were participants are able to define the activation and deactivation time of a device. Results showed reduced energy consumption to groups with online controls compared to just feedback. Lights and phone devices showed higher savings compared to computer and monitors. Automation control was less effective to users with an ingrained energy efficient behaviour.

Games have also been used with the aim of promoting proenvironmental behaviour, mostly in the domestic context. For example, Reeves et al. [16] presented a serious game, Power House, that simulates a virtual household and evaluated its consequence both in a lab study and in a field trial, reporting its success in the reduction of energy consumption. Few energy related games were specifically targeted to children and adolescents. Gustafsson et al. [7] argue that serious games make it difficult to transfer lessons from the game to the real world, therefore they propose instead a casual game approach. They report the design of a pervasive game for teenagers, played by activating and deactivating real appliances in the home (monitored through plug-level sensors). A similar approach is proposed by Bang et al. [1], who report a combination of a casual game, that follows a classic videogame gameplay, with a pervasive game in which players have to complete missions in the real world and verify their action by taking pictures with smart phones. The game aims to educate teenagers on ways to conserve energy at home, but no user evaluation is reported. In contrast to this prior work, IdleWars, the game we present here, is a pervasive game designed for adults in the work environment.

The only game designed to encourage proenvironmental behaviour in the work environment is Climate Race [20], which is based on a combination of implicit and explicit energy-related actions. The game tracks players' activity in the real world at the room level, through environmental sensors (e.g., switching off lights when not in the office); based on this activity players gain positive or negative points. Extra game points can be collected through specific collective actions, such as all players switching off lights when leaving the office. Our approach is different from Climate Race in two ways: first, it does not require sensors, as activity detection takes place in software on existing office IT infrastructure. Second, IdleWars, introduces an element of competition its the game dynamics, with the aim to increase engagement.

The idea of using computer idle time as a proxy for energy wasteful behaviour was originally proposed by Kim et al. [13], who used this measure to investigate two persuasive ambient displays: Timelog and Coralog. Both visualisations aim to eliminate computer idle time; Timelog uses a bar graph to represent the active and idle time of the computer, while Coralog uses a visual metaphor, where the proenvironmental behaviour is mapped to the health of coral reefs. Through a study, Kim et al. showed that the Coralog visualisation created emotional attachment and the desire to change behaviour 


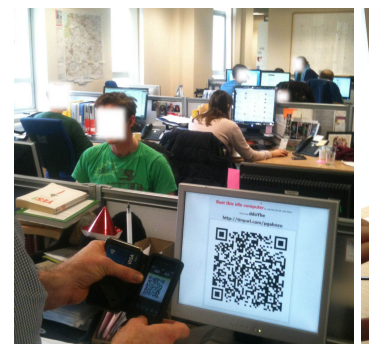

(a)

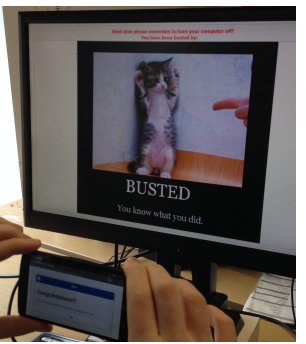

(b)

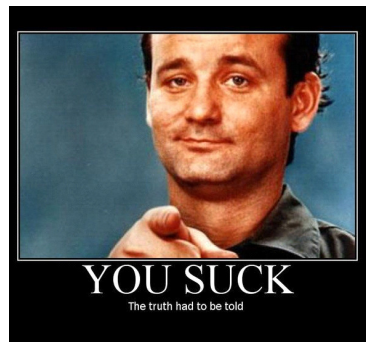

(c)

Figure 1: (a) A participant busting the idle computer of another player by scanning the QRCode on the IdleWars screen saver. (b) A busted computer showing the profile picture of the player. (c) The profile image used by a participant to convey a message to players they bust.

whereas the one based on bar charts did not. While the work by Kim et al. focusses on individual users, IdleWars uses computer idle time in the context of a game and the online nature of it provides not only personal feedback, but also comparative feedback, as detailed next.

\section{Game Design and its Rationale}

We started the design process by taking into account the main contrasts between the workplace and domestic environments, to try and best apply lessons from prior work. The first important difference is the lack of incentives: employees generally do not share financial benefits coming from lower energy bills $[8,4,9]$. Another key difference is that workplaces often have a richer social dimension than in a domestic context, not only because generally there are more people in an office than a home, but also because these multiple social groups and layers (e.g. friends, teams, divisions, departments, crosscutting projects, etc.) may co-exist among workers.

Against this background, we decided to design a game. We believe that through a balance of competition and collaboration games have potential to leverage and influence social dynamics, in a way that can be steered towards proenvironmental behaviour. Moreover, it was recently reported that games in the workplace have potential to provide motivation for employees to reduce their energy consumption [20]. We decided to focus on wastage around personal computer usage for several reasons: first, in the work environment the computer is mostly a personal tool and only its owner has the responsibility of switching it on and off, so it is possible and easy to track individual behaviour, in contrast to shared equipment (e.g. from shared printers to coffee machines to corridor lights), for which apportionment would be more difficult or even impossible. Second, monitoring the PC can be achieved purely in software, without any additional hardware, therefore keeping deployment costs and installation complexity low and making the system easily scalable. 
IdleWars, the game we designed, tracks the computer status for each player. When no mouse movements or key strokes are detected for more than 5 minutes, the computer is considered inactive, or "idle". In such case, a screensaver appears on the computer screen, showing a QR code, a short url, and an additional alphanumeric code, as illustrated in Figure 1a. Any player (other than the computer owner), can then "bust" the idle computer by scanning the QR code with a smartphone, or by manually typing the short url or the alphanumeric code in any web browser (in case a smart phone is not available). Following the busting action, the screensaver of the idle computer changes to show the profile picture of the person who busted the computer, as illustrated in Figure 1b. At any point the "owner" of an idle computer, whether busted or not, can close the screensaver and resume the normal operation by typing in their password. If the idle computer is busted, the owner will see a full-screen profile picture of the player who busted them when they return to their desk. Once a computer has been busted by one player, it cannot be busted by anyone else.

Busting an idle computer can be considered as a metaphor for turning it off. Therefore, busting is a proenvironmental, energy-saving behaviour that we want to encourage through the game. Conversely, leaving a computer idle represents a wasteful behaviour, which in our game makes the player vulnerable to being busted by other players. The system tracks the time (in minutes) that a computer stays busted - this time is roughly related to the amount of energy that would have been saved by switching the computer off. This information is considered eco-feedback on the premise of reducing environmental impact [5]. The total time busted (which can be considered "time rescued") by each participant, the total number of busting actions, and the percentage of individual idle time are used to calculate 3 separate player rankings displayed on the IdleWars leader board, as shown in Figure 2. All metrics and ranking orientations are designed to give emphasis to positive behaviour (rather than highlighting negative behaviour).

The leader board provides players with comparative and continuous feedback about their behaviour. It is displayed on a public screen in the workplace where the game takes place, and it is also accessible as a Web page. Users can only access the Web page version by logging-in, which allows us to highlight the individual player position in each of the rankings, making it easier to read. The public display was deliberately designed not to be interactive, so it does not support scrolling, and it shows only the top performers from each ranking. The number of top participants shown depends on the size of the screen available for deployment. The choice of a public display, to be placed in a trafficked location in the workplace, aims at encouraging casual conversations and triggering gossip related to the game, with the hope to further motivate workers towards the desired behaviour.

As privacy was reported to be an issue of concern in the work environment $[8,20]$, in IdleWars the idle time is presented in terms of percentage of the total time the computer is on. In this way the information about the total time each computer is active or idle is kept private. Another major concern in a work environment is productivity: the game must not obstruct employees. For this reason IdleWars does not implement any notification mechanism, which may be considered distracting and disruptive. Feedback is provided only through the leader board, and through the game screensaver indicating that the computer was left idle wasting energy, which acts as an ambient display. 


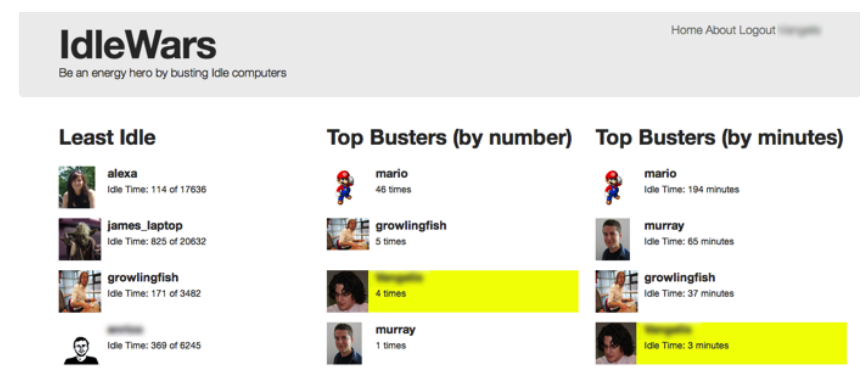

Figure 2: The IdleWars leader board.

\section{Deployment}

We conducted an initial deployment to assess whether the game dynamics would engage people, and to observe whether any changes would occur in terms of computer IdleTime. The trial lasted for two weeks, and it took place at the Centre for Sustainable Energy (CSE), a non-profit organisation working on sustainable energy \& policy. The organisation has 50 employees, most of them located in one workspace: an office including two communicating large open spaces, see Figure 1a for a partial view. This setup allows each player to easily see and scan the computer screen of other workers. Computer usage is an important part of the office work, main activities are e-mail, writing reports and searching the Web for information. At one end of the working space there is an open plan kitchen, used to warm up and consume meals and to make hot drinks. The leader board semi-public display was installed in front of the kitchen, to make it visible and encourage people to talk about the game and the ranking over lunch and coffee breaks.

The trial was approved by the organisation's management, and recruitment took place through an email sent to all employees, and through an announcement at a staff meeting. Participants were asked to register on a website, and at the same time provide consent to participate in the research. The game software client could also be downloaded from the site, at the end of the registration process. An experimenter assisted participants in the installation process.

Automatic interaction logs were collected throughout the duration of the trial. After the end of the trial we conducted a focus group. The format of a focus group was chosen, rather than individual interviews, due to the very limited time availability of participants. The session took place over a lunch break and it lasted approximately one hour.

A total of 26 participants ( 15 females) registered but only 20 (11 females) installed and used the system. All participants are staff members of the organisation. They all hold a degree level and some have post-graduate qualifications. Ages range from late 20 s to early 40 s, with most in their 30s. 7 of the players took part in the focus group, together with one employee who did not play but expressed interest. 


\section{Findings}

We report findings from the focus group (through thematic analysis [3]) and present information on system usage based on the automatic interaction logs. The focus group was audiorecorded and transcribed for the analysis.

\subsection{Interaction logs}

Interaction logs were automatically collected by the system, including: idle and active time, bust attempts, and web page views. During the 10 working days period, computers were left idle for 3719 minutes overall, corresponding to $8.1 \%$ of the total time they were on. If busting a computer represented shutting it down, participants would have saved 268 minutes of computer idle time, corresponding to $6.7 \%$ of the total idle time.

In total, 14 participants out of 20 busted a computer at least once. Most activity happened during the first week with 23 busting actions, whereas in the second week only 14 took place. We found that the total 37 busting actions took place on just 9 computers, which got busted from 2 to 11 times, while the remaining computers were never busted. All 7 participants who joined the focus group were involved in the actual game play, in the sense that they either busted or got busted 3 or more times.

\subsection{Engagement}

The focus group revealed enthusiastic engagement with the game. Participants reported running and having fun, for example: "Yes. There was a lot of noise when P4 was sprinting across the office, shouting 'no!' [because his computer was about get busted by another participant]" [P6]. 'Fun' was also mentioned explicitly: “...you know, it's quite fun to have someone's profile picture coming up as Bill Murray saying 'you suck'." [P4] This comment refers to the profile image used by another participant, shown in Figure 1c. Indeed, another sign of engagement was the appropriation around the use of profile images. While we suggested participants to use an image to represent them (an avatar), three of them chose instead an image with humorous text (a so called "image macro" in Internet slang). This is because these participants realised that the profile picture would be displayed on the screen of a busted computer, so they used it to deliver a message to the people they bust. This practice was widely accepted and characterised as fun by the participants, as the previous quote illustrates.

Apparently, our participants became so engaged that game-related tension mounted around the risk of having one's computer busted: “...it became quite a tense office, because if anybody did leave their desk and left it [the computer] on, there'd be quite a few people around it just... waiting." [P5] This quote also indicates the development of tactics, such as players paying attention to who gets up from the desk. Another participant also described a similar tactic, to see who is in the kitchen (which is part of the office open plan) and then check whether their computers are idle: "If you keep an eye on the kitchen... see who was in the kitchen, and then go and look at their desk." [P1]

In contrast to the above report of the game generating 'tension' someone else told us that the game also had a stress relieving effect: "I think particularly because we have 
got lots of work on at the moment, it's always nice to have something.. ..stop you [from] stressing." [P7] The game, then, acted as a welcomed distraction from everyday issues.

To sum up, many of the comments from the focus group provide an indication of how engaging the game was and how the work environment became a more active place relieving workers from stress. The focus group also reveals how participants devised new ways of using the system to interact with each other and tactics on how to score more.

\subsection{Gameplay}

From the focus group it became apparent that during the game participants viewed the number of times they busted other players as a score for the game. They placed less value in the minutes they "rescued" by busting others, or in limiting the minutes their computer was idle (as described in the Game Design section). One participant explained that the number of times they busted others was perceived as a metric for one's own more "active" gameplay, while the number of minutes busted depended more on others' behaviour:

"I think there's inherently a bit more glory within sort of the number of times that you've busted, [...] because it's quite arbitrary, how long it takes somebody to come back to their computer. It's not like that's your victory as a buster.." [P5]

Another participant reinforced the idea: "I looked at that [the idle time] briefly, but I suppose... not such an interesting bit, for me. It was more the action [of busting] that was the interesting bit." [P6] Participants also reported a strategy to increase the number of times they busted others: after busting the computer of someone who was away from their desk, they would then unlock the computer and wait for it to become idle again after five minutes, so it could be busted one more time.

Someone else related the busting action to direct competition: "Well, there's the point of the busting, yes [...] You can sort of say, oh, 2-0 or whatever. It's easy to compare." [P2] This quote, as well as several other comments made throughout the focus group, suggest that our participants were very sensitive to the competitive aspect of the game.

At the same time, another participant highlighted a conflict between competitive, individualistic behaviour and sustainability:

"I think I have a bit of a thing about this ... if you encourage people to take a competitive, individualistic approach, you're kind of encouraging them to behave in a particular way which actually, in a holistic sense, isn't that good for being sustainable. So kind of bringing out certain characteristics of them ...” [P1]

We also learned that another co-worker, who did not take part in the focus group, declined to take part in the game because they disliked this mismatch between sustainability and competition: "There was somebody who didn't play out of principle, because they thought it shouldn't become a competitive [activity]" [P1]

In summary, the gameplay was dominated by the number of times participants busted others, which aligned with the competitive attitude most participants had during the game. However, some participants called attention to the contrast between individualistic attitude typical of competition and sustainable behaviour. 


\subsection{Awareness and Behaviour Change}

The game triggered a discussion in the workplace about computer power management, as that was perceived to be directly related to energy waste. Participants realised that they could save energy by deactivating their computers in different ways: "We had a discussion about what the difference between hibernation and sleep was, didn't we? And somebody broke down which one was better. Mark did some sums" [P7]

The influence of the game extended even to those in the office who did not participate in the game. We received indirect reports that even those not playing the game felt more aware of their behaviour around energy waste, because of having people participating around them.

The discussion also highlighted technical issues related to computer power management:

"...it took so long to come back up if you hibernated your computer. So I think maybe it's a bit unrealistic to tell people they need to hibernate, because if you're away for five minutes, that's a bit of a pain.." [P1]

Other participants mentioned that different computers (running different versions of the operating system) had different power management options and different problems. For example, some applications would not reconnect to their servers after computers were resumed from sleep or hibernation.

Moreover, the focus group revealed that the game had also undesirable effects on power management. It turned out that setting computers to automatically sleep or hibernate after 5 minutes was considered as "cheating" in the context of the game. One of the participants told us: "I did [configure my computer to automatically hibernate], and then I got pressured that I was cheating, and then reverted back." [P4] another one confirmed: "It's no fun [to configure your computer to automatically hibernate]. That's the thing? it was no fun if anyone was able to do that." [P6] Configuring the computer to automatically hibernate was deemed not acceptable by the rest of the participants, because it would take the fun of busting away. As P6 explains, if everyone activates this automation none of the computers will become idle and therefore it would become impossible to play the game.

The negative effects of the game on power management went even further. Some of the participants had the habit of switching their monitor off (albeit not the computer) when leaving their desk. However, this would make it impossible for other players to bust the computer, so they were pressured into foregoing this habit:

"P6: I think we're all in the habit of just turning off our monitors. So you had to undo that, because really we are used to turn off the monitors.

P4: ..To enjoy the game."

The discussion stimulated by IdleWars extended beyond energy consumed by computers, to a more general level. In part this generalisation was prompted by the understanding that computer consumption could be quite minimal:

"Is the expectation that the benefit will come on saving energy for the monitors, or is it from the kind of discussion that might happen around it? [...] Because actually, the amount, you know, we would have saved is vanishingly small, presumably, isn't it?" [P2] 
So participants also considered energy waste, automation and behaviour change related to other office appliances, such as shared printers, or lights, as demonstrated by the following exchange:

"P4: We don't switch the lights off [...]. I used to always do it... the ones in the kitchen. I gave it up. They never get done.

P7: Are they not motion-sensitive?

P4: No. No, you can switch them [the lights] off. So you' re right... you know, we don't do things we could do."

As such, while the IdleWars game stimulated participants to forego proenvironmental behaviours such as setting computers to automatically hibernate or sleep or turning off monitors when not in use, it also raised awareness around energy waste more generally in the workplace, and encouraged the players to reflect on a broader array of energy consuming behaviours.

\section{Discussion}

The game design was successful in engaging participants, as demonstrated by the interaction logs, by the focus group and by the appropriation of the profile images. IdleWars sparked discussion around energy waste and conservation: participants explored different options for computer power management (sleep and hibernation) they had not considered before, and confronted their shortcomings. The sleep and hibernation shortcomings can potentially be diminished by the adoption of new hardware and software technologies (e.g. faster hard-disk drives). At the same time, the game turned out also to encourage some anti-conservation behaviours: discouraging users from automatically setting their computers to sleep and from turning monitors off. It is worth emphasising, then, that the game was effective in terms of generating discussion and even behaviour change, yet not necessarily for the better.

Despite the engagement, though, we could not find statistically significant differences in idle time and indeed a reduction in idle time of 5.6\% is quite low in absolute terms. This could be explained by the combination of proenvironmental and wasteful behaviours that were encouraged by the game, as well as by a strong proenvironmental culture in the organisation where the game was deployed. Indeed, a computer idle time of only $8.2 \%$ is quite low, and it is clear from the logs that none of the participants left their computer on overnight.

More specifically, the game design was successful in catalysing existing social dynamics in the work environment where it was deployed: our participants collectively interpreted how the game was supposed to be played, to the point of making up additional rules (e.g. it is forbidden to automatically put one's computer to sleep). The main implication of these findings, we argue, is that they demonstrate the potential of games in the workplace to engage workers around sustainability issues, to stimulate discussion, and even encourage behaviour change.

\subsection{Physicality and Visibility}

Based on the focus group, the main factors behind the success of the game in engaging participants seem to be its physical elements and its competitive nature. The physicality 
contributed to make the gameplay visible. Participants saw others "sprint" across the office to save their computer from being busted. Scanning a QR-code to bust a computer is a gesture that everyone in the office can see. The idle and busted screen-savers as well as the leader board are visible in the workplace, making everyone aware of the status and activity of everyone else. The IdleWars leader board also made players' behaviour visible, revealing the proenvironmental or wasteful behaviour of the individual, and potentially even the proportion of time one spends at their desk.

These results, then, bear an implication for future research, opening up a question about how similar visibility could be achieved at a larger scale. Would it be possible to make this type of games work at all, for example, in larger companies, where teams are not co-located? Further research could explore the application of remote collaboration paradigms, such as ambient displays that show when a remotely located computer becomes idle and then gets busted.

\subsection{Action-Reaction in the Gameplay}

Being "active" seems to be a key for our participants. They found the idea of gaining points for busting someone else rewarding because they relate it directly to the prowess of the buster. In contrast, gaining more or less points because the person busted left their computer inactive for a particular length of time was perceived as depending (somewhat arbitrarily) on the fault of the another person, and therefore not of interest. Similarly, setting computers to automatically sleep after few minutes of inactivity was considered cheating - it is an individual responsibility, one needs to remember to turn off the computer, so they can be caught if they forget. As such, automation was found not to be fun . This effect is perhaps encouraged or amplified by the feedback provided by IdleWars. The action of busting a player is instantly rewarded by the feedback of having one's profile picture displayed on the screen of the "victim". The appropriation we observed around the use of the profile pictures further indicates that our participants valued this action-reaction sequence.

The lack of interest in the number of minutes "rescued" by busting can also be explained due to the fact that IdleWars does not provide instant feedback about the minutes busted: players need to go and find the leader board to reveal how many minutes the busted player had been idle for. Simply adding a minutes counter on the busted screen would make this information instantly available, and thus more salient to the players. On the other hand, a busted computer could be unlocked by anyone and busted again after five minutes. This made it possible, and even encouraged, to score repeatedly. This issue could be limited by making the unlocking of a busted computer password-protected, so that only the computer owner could perform it. This shortcoming negatively influenced the game dynamics. One strategy to limit this type of issue could be, for example, to include in the game explicit suggestions about proenvironmental behaviour, and perhaps even to make the action of busting a computer metaphor for switching it off.

At one level, it could be argued that the undesired effects were simply caused by a design limitation, which resulted in a misalignment between the (perceived) game goals and the desired behaviour. However, more in general, it is worth calling attention to the potential conflict between individualist competition and sustainability goals, often framed in terms of altruistic and cooperative behaviour. Indeed, at least one worker 
from the organisation where we deployed the game refused to take part because she felt the two attitudes should not be combined. This question highlights an opportunity for further research.

\subsection{Productivity Trade-offs?}

Similar to other studies about energy conservation in the workplace [12], a tension between saving energy and productivity on the job emerged in our focus group. IdleWars encouraged our participants to put their computer to sleep or into hibernation, but they perceived that such practice has the potential to reduce their productivity, because it takes time to reactivate the computer and resume work when one is back at the desk, or because of software glitches. Some of these issues are strictly technological (rather than behavioural), and probably related to dated software and hardware. While hardware upgrade is likely to have a considerable environmental cost, a purely software solution (e.g. having applications that reconnect to servers in seamless fashion after computer sleep) could be attractive, if at all possible [2].

The IdleWars gameplay in itself was also pointed out to be a source of distraction: a few keen players admitted they would sometimes linger away from their desk to try and bust others, or they would run and shout in the office distracting bystanders. These are probably extreme cases, and indeed the reports from other participants suggest the gameplay was often integrated in the natural work breaks that take place in any workplace. However, we believe these occurrences point at another inherent tension: between job productivity and an engaging, entertaining game.

We draw two implications here. First, to contain the distraction caused by games like IdleWars, more efforts could be made to refine their design, using timed activation to fit within prescribed pauses, or limiting the daily amount of playing. Second, given that the aim is to help players learn a proenvironmental behaviour, an alternative strategy could be to frame such games as episodic, short term activities lasting just one or two weeks. The game could then become one of a number of activities (e.g. workshops) designed to draw employees attention to sustainability issues in the workplace, all to take place over a specific period. Creating anticipation for the event, by advertising it in advance, could help the engagement, as it happened in our deployment. This duration-limited approach would also be inline with the engagement naturally tapering off over time.

\section{Conclusion and future work}

In this paper we introduced IdleWars, a pervasive game designed to raise awareness and promote behaviour change in relation to energy waste in the workplace. An initial deployment, over two weeks in a medium sized organisation, revealed that the physical and competitive elements of the game work well in engaging participants. More specifically, the design was successful in catalysing existing social dynamics in the workplace where it was deployed. 
Participants appropriated the game and extended its rules, sometimes in a way that favoured engagement and fun rather than conservation behaviour. IdleWars triggered discussion around computer power management options and their adoption, and more in general on energy waste in the office. In contrast, setting computers to automatically sleep after few minutes of inactivity (which is desirable in terms of sustainability) was considered "cheating" because it takes away from the game challenge. While these results point out that our specific game design needs to be revised to better align the game rules with the underlying sustainability goals, they also indicate that pervasive games like IdleWars can be effective tools to raise the attention to sustainability issues in the workplace, paving the way for further HCI research in this domain.

In addition to the pointers included in the discussion, in future work we also plan to leverage the IdleWars infrastructure, especially in terms of idle time sensing, with alternative game or eco-feedback approaches. We believe that computer idle time as a measure of proenvironmental behaviour has potential for larger scale, remote deployments, and engagement through online social networks.

Acknowledgments This work was partially supported by the "Creating the Energy for Change" project (energyforchange.ac.uk) reference No. (EP/K002589/1) and by the Greek State Scholarships Foundation (www . iky • gr), contract No. (2012ПЕ-564). We would like to thank the Centre for Sustainable Energy (www. cse. org . $u \mathrm{k}$ ) for allowing us to trial our prototype at their premises. The dataset is available at (http://dx.doi.org/10.5258/SOTON/377465)

\section{References}

1. Bang, M., Gustafsson, A., Katzeff, C.: Promoting New Patterns in Household Energy Consumption with Pervasive Learning Games. In: Kort, Y., IJsselsteijn, W., Midden, C., Eggen, B., Fogg, B. (eds.) Persuasive Technology, Lecture Notes in Computer Science, vol. 4744, pp. 55-63. Springer Berlin Heidelberg (2007)

2. Blevis, E.: Sustainable Interaction Design: Invention \& Disposal, Renewal \& Reuse. In: Proc. CHI'07 (2007)

3. Braun, V., Clarke, V.: Using thematic analysis in psychology. Qual. Res. in Psych 3(3), 77102 (2006)

4. Foster, D., Lawson, S., Wardman, J., Blythe, M., Linehan, C.: "Watts in It for Me": Design Implications for Implementing Effective Energy Interventions in Organisations. In: Proceedings of the SIGCHI Conference on Human Factors in Computing Systems. pp. 2357-2366. CHI '12, ACM, New York, NY, USA (2012)

5. Froehlich, J., Findlater, L., Landay, J.: The Design of Eco-feedback Technology. In: Proceedings of the SIGCHI Conference on Human Factors in Computing Systems. pp. 1999-2008. CHI '10, ACM, New York, NY, USA (2010)

6. Gaver, W.W., Bowers, J., Boucher, A., Gellerson, H., Pennington, S., Schmidt, A., Steed, A., Villars, N., Walker, B.: The Drift Table: Designing for Ludic Engagement. In: CHI '04 Extended Abstracts on Human Factors in Computing Systems. pp. 885-900. CHI EA '04, ACM, New York, NY, USA (2004)

7. Gustafsson, A., Bång, M., Svahn, M.: Power Explorer: A Casual Game Style for Encouraging Long Term Behavior Change Among Teenagers. In: Proceedings of the International 
Conference on Advances in Computer Enterntainment Technology. pp. 182-189. ACE '09, ACM, New York, NY, USA (2009)

8. Jahn, M., Schwartz, T., Simon, J., Jentsch, M.: EnergyPULSE: Tracking Sustainable Behavior in Office Environments. In: Proceedings of the 2nd International Conference on EnergyEfficient Computing and Networking. pp. 87-96. ACM Press, New York, New York, USA (2011)

9. Jain, M., Agrawal, A., Ghai, S.K., Truong, K.N., Seetharam, D.P.: "We are not in the loop": Resource Wastage and Conservation Attitude of Employees in Indian Workplace. In: Proceedings of the 2013 ACM international joint conference on Pervasive and ubiquitous computing. pp. 687-696. UbiComp '13, ACM, New York, NY, USA (2013)

10. Jain, R.K., Taylor, J.E., Peschiera, G.: Assessing eco-feedback interface usage and design to drive energy efficiency in buildings. Energy and Buildings 48, 8-17 (may 2012)

11. Jentsch, M., Jahn, M., Pramudianto, F., Simon, J., Al-Akkad, A.: An Energy-Saving Support System for Office Environments. In: Salah, A., Lepri, B. (eds.) Human Behavior Understanding, Lecture Notes in Computer Science, vol. 7065, pp. 83-92. Springer Berlin Heidelberg (2011)

12. Katzeff, C., Broms, L., Jönsson, L., Westholm, U., Räsänen, M.: Exploring Sustainable Practices in Workplace Settings Through Visualizing Electricity Consumption. TOCHI 20(5) (Nov 2013)

13. Kim, T., Hong, H., Magerko, B.: Design Requirements for Ambient Display That Supports Sustainable Lifestyle. In: Proceedings of the 8th ACM Conference on Designing Interactive Systems. pp. 103-112. DIS '10, ACM, New York, NY, USA (2010)

14. PC Energy Report: PC Energy Report 2009. http://www.1e.com/ energycampaign/downloads/PC_EnergyReport2009-US.pdf (2009), Accessed: 2013-09-12

15. Pousman, Z., Rouzati, H., Stasko, J.: Imprint, a Community Visualization of Printer Data. In: Proceedings of the ACM 2008 conference on Computer supported cooperative work CSCW '08. pp. 13-16. ACM, San Diego, CA, USA (2008)

16. Reeves, B., Cummings, J.J., Scarborough, J.K., Yeykelis, L.: Increasing Energy Efficiency With Entertainment Media: An Experimental and Field Test of the Influence of a Social Game on Performance of Energy Behaviors. Environment and Behavior (2013)

17. Ryan, R.M., Deci, E.L.: Intrinsic and Extrinsic Motivations: Classic Definitions and New Directions . Contemporary Educational Psychology 25(1), 54 - 67 (2000)

18. Schwartz, T., Betz, M., Ramirez, L., Stevens, G.: Sustainable Energy Practices at Work: Understanding the Role of Workers in Energy Conservation. In: Proceedings of the 6th Nordic Conference on Human-Computer Interaction: Extending Boundaries. pp. 452-462. NordiCHI '10, ACM, New York, NY, USA (2010)

19. Siero, F.W., Bakker, A.B., Dekker, G.B., Van den Burg, M.T.: Changing Organizational Energy Consumption Behaviour through Comparative Feedback. Journal of Environmental Psychology pp. 235-246 (1996)

20. Simon, J., Jahn, M., Al-Akkad, A.: Saving Energy at Work: The Design of a Pervasive Game for Office Spaces. In: Proceedings of the 11th International Conference on Mobile and Ubiquitous Multimedia. pp. 9:1-9:4. MUM '12, ACM, New York, NY, USA (2012)

21. Yun, R., Aziz, A., Scupelli, P., Lasternas, B., Zhang, C., Loftness, V.: Beyond Eco-Feedback: Adding Online Manual and Automated Controls to Promote Workplace Sustainability. In: Proceedings of the 33rd Annual ACM Conference on Human Factors in Computing Systems. pp. 1989-1992. CHI '15, ACM, New York, NY, USA (2015) 